\title{
Valor pronóstico de los anticuerpos anti-Chlamydia pneumoniae en la cardiopatía isquémica
}

\author{
C. González Castañeda, J. L. Pérez Castrillón, A. Casero Lambas, M. Á. Mazón Ramos, \\ M. Á. Cañibano González, M. López Bello y V. Herreros Fernández \\ Servicios de Medicina Interna y Cardiología. Hospital Universitario Río Hortega. Universidad de Valladolid. Valladolid.
}

\begin{abstract}
Introducción y objetivos. Se ha relacionado la enfermedad arterial coronaria con infección por Chlamydia pneumoniae, pero existen pocos estudios que valoren la presencia de anticuerpos frente a este microorganismo y el pronóstico de los pacientes con cardiopatía isquémica. El objetivo de nuestro estudio fue valorar el impacto de la positividad de anticuerpos anti-Chlamydia pneumoniae en la morbimortalidad asociada a la cardiopatía isquémica. Métodos. Se estudiaron 249 pacientes con cardiopatía isquémica (97 con infarto de miocardio, 83 con angina inestable y 69 con angina estable) recogidos durante un año y seguidos tres años. Se determinaron anticuerpos IgG anti-Chlamydia pneumoniae por la técnica de microinmunofluorescencia. El punto de corte considerado como serología positiva lo establecimos en $1 / 64$.

Resultados. En el grupo de 97 pacientes con infarto agudo de miocardio, un $43 \%$ tenía títulos positivos de anticuerpos IgG frente a $C$. pneumoniae. Observamos asociación estadísticamente significativa $(p=0,007)$ entre la serología positiva y la mayor supervivencia de los pacientes con infarto agudo de miocardio. Se realizó una regresión logística considerando mortalidad, edad y serología siendo la edad la única variable que explicaba la mortalidad, con una $\mathbf{p}=\mathbf{0 , 0 0 1 2}$, perdiendo la serología su significado. En el grupo de 83 pacientes con ángor inestable y de 69 pacientes con ángor estable, el $42 \%$ y el $58 \%$ respectivamente, tenía serología positiva a C. pneumoniae. En ambos grupos no encontramos asociación estadísticamente significativa entre la serología positiva a $C$. pneumoniae y los factores pronósticos de morbimortalidad (reingresos y mortalidad).

Conclusiones. La presencia de anticuerpos IgG frente a $C$. pneumoniae no es un marcador de morbimortalidad en nuestra población con cardiopatía isquémica.
\end{abstract}

PALABRAS CLAVE: Chlamydia pneumoniae, morbimortalidad, cardiopatía isquémica.

González-Casteñeda C, Pérez-Castrillón JL, Casero-Lambas A, Mazón-Ramos MÁ, Cañibano-González MÁ, López-Bello M, Herreros-Fernández V. Valor pronóstico de los anticuerpos antiChlamydia pneumoniae en la cardiopatía isquémica. Rev Clin Esp 2004;204(11):583-7.
Prognostic value of antibodies anti-Chlamydia pneumoniae in ischemic heart disease

Introduction and objectives. Coronary artery disease has been related to infection by Chlamydia pneumoniae even only in a few studies the association between presence of antibodies to this microorganism and the prognosis of patients with ischemic heart disease has been assessed. The objective of our study was to assess the impact of positivity of anti-Chlamydia pneumoniae antibodies in the morbidity and mortality associated to ischemic heart disease.

Methods. 249 patients with ischemic cardiopathy were evaluated (97 with myocardial infarction, 83 with unstable angina and 69 with stable angina), recluted along 1 year and with 3-year follow-up. IgG anti-Chlamydia pneumoniae antibodies were measured with microimmunofluorescence. The cut off point considered for positive serology was set in $1 / 64$.

Results. In the group of 97 patients with acute myocardial infarction, $43 \%$ showed positive titers of IgG antibody to $C$. pneumoniae.

We observed a statistically significant association $(p=0.007)$ between positive serology and higher survival of acute myocardial infarction patients. A logistic regression was carried out with mortality, age, and serology as variables; age was the only variable that explained mortality $(p=0.0012)$, and the serology lost its statistical meaning. In the groups of 83 patients with unstable angina and of 69 patients with stable angina, $42 \%$ and $58 \%$, respectively, showed positive serology for C. pneumoniae. We did not find a statistically significant association in both groups between positive serology to $C$. pneumoniae and the prognostic factors related to morbidity and mortality (rehospitalization and mortality). Conclusions. The presence of IgG antibodies to C. pneumoniae is not a marker of morbidity and mortality in our population of patients with ischemic heart disease.

KEY WORDS: Chlamydia pneumoniae, morbidity, mortality, ischemic heart disease.

\section{Introducción}

La aterosclerosis es la mayor causa de morbimortalidad en el mundo occidental. Los factores de riesgo 
cardiovascular conocidos (tabaco, diabetes, hipertensión arterial y dislipemia) no explican completamente las variaciones temporales y geográficas en la prevalencia de la enfermedad cardiovascular ${ }^{1}$.

Estudios recientes han mostrado que algunos pacientes sin factores de riesgo clásicos tenían evidencia de una respuesta inflamatoria exagerada que podría desencadenar oclusión vascular. Signos de este proceso inflamatorio, como aumento de la velocidad de sedimentación globular, de proteína $C$ reactiva y proteína amiloide A, se han encontrado en pacientes con infarto agudo de miocardio y angina inestable, así como, en esta última, presencia de linfocitos $T$ activados circulantes, como indicador de estimulación antigénica que es parte de la respuesta inflamatoria. Recientemente se ha incriminado a microorganismos como "antígenos desencadenantes" de esta reacción ${ }^{2}$.

Se ha mostrado la relación existente entre la incidencia de aterosclerosis y la presencia de microorganismos, entre ellos la Chlamydia pneumoniae. En 1988, Saikku detectó que los pacientes con infarto agudo de miocardio y ángor pectoris tenían una significativa elevación de los títulos de anticuerpos anti-Chlamydia pneumoniae (inmunoglobulina $\mathrm{G}[\mathrm{IgG}]>1 / 128$ ) frente a controles asintomáticos ${ }^{3,4}$. Hay diversos estudios patológicos y seroepidemiológicos que han observado la existencia de una asociación positiva entre infección pasada o actual por C. pneumoniae y enfermedad arterial coronaria, llegando en algún caso a cultivarse C. pneumoniae viable de tejido aterosclerótico. Otros estudios detectaron frecuentemente C. pneumoniae en las placas ateromatosas de arterias coronarias; sin embargo, su distribución no se correlaciona con la severidad o extensión de la enfermedad ${ }^{5}$, pero apoyaría la hipótesis de que el organismo puede ser un factor activo en la patogénesis de la aterosclerosis ${ }^{6}$. Sin embargo, existen pocos estudios, ninguno realizado en España, que determinen el valor pronóstico de la presencia de anticuerpos frente a C. pneumoniae en pacientes con cardiopatía isquémica. El objetivo de nuestro estudio fue valorar el impacto de la positividad de anticuerpos anti-C. pneumoniae en la morbimortalidad cardiovascular asociada a la cardiopatía isquémica.

\section{Material y métodos}

\section{Material}

Nuestro estudio fue de cohortes prospectivo y la población estudiada incluyó 249 pacientes con cardiopatía isquémica, separándolos en tres subgrupos: 97 con infarto de miocardio, 83 con angina inestable y 69 pacientes con angina estable que fueron recogidos en nuestro hospital durante el período de un año, desde mayo de 1997 hasta mayo de 1998; 174 eran varones (70 con infarto de miocardio, 55 con angina inestable y 49 con angina estable) y 76 mujeres (27 con infarto de miocardio, 29 con angina inestable y 20 con angina estable); 79 procedían del ámbito rural y $170 \mathrm{del}$ urbano; la edad estaba comprendida entre los 31 y los 94 años, con una edad media de $67,9 \pm 12,1$ años. De los subgrupos con angina inestable e infarto agudo de miocardio, se recogió inmediatamente después del ingreso con el episodio coronario agudo una muestra de sangre para deter- minación de anticuerpos frente a C. pneumoniae, en el subgrupo con angina estable se citó a los pacientes, previamente diagnosticados, mediante envío de una carta a su domicilio donde se les pedía colaboración voluntaria para la realización de la extracción de la muestra sanguínea. Posteriormente los pacientes fueron seguidos hasta un período máximo de tres años.

Los pacientes fueron diagnosticados de infarto agudo de miocardio, ángor estable e inestable, de acuerdo con los criterios de la Sociedad Española de Cardiología?

\section{Definición de casos}

1) Infarto agudo de miocardio. Presencia de al menos dos de los siguientes criterios: a) dolor anginoso de > de $30 \mathrm{mi}-$ nutos de duración; b) elevación de las concentraciones de enzimas usadas para el diagnóstico de infarto agudo de miocardio (creatincinasa [CK]) al menos al doble de los valores normales, y c) elevación del ST > $1 \mathrm{~mm}$ en al menos dos derivaciones estándar o en tres precordiales, o desarrollo de ondas Q en el electrocardiograma (ECG).

Se incluyeron pacientes con y sin tratamiento trombolítico. 2) Angina estable. Dolor, opresión o malestar localizado en el área esternal, irradiado a hombros y brazos; b) desencadenado habitualmente por el ejercicio cediendo con el reposo o tras administración de nitroglicerina sublingual; c) la duración del dolor es de unos minutos; raramente dura menos de un minuto; d) no presenta cambios en su patrón en el último mes de evolución, y e) cursa con alteraciones de la repolarización ventricular en el ECG, siendo la más frecuente la infradesnivelación del segmento ST.

3) Ángor inestable. Dolor debido a isquemia miocárdica, que requiere hospitalización por dificultad en su control o para descartar que sea debido a un infarto agudo de miocardio. Incluye: a) angina crónica que aumenta su intensidad, frecuencia o duración; b) aparición de angina de reposo, y c) angina grave de reciente inicio.

\section{Métodos}

En suero de los pacientes se determinaron anticuerpos IgG anti-C. pneumoniae utilizando la técnica de microinmunofluorescencia (MIF). La prueba utilizada fue la de MRL Diagnostics (California, USA). Emplea como antígeno el lipopolisacárido de membrana externa correspondiente a la cepa TW183. Permite diferenciar la infección por C. pneumoniae de infecciones por otros tipos de Chlamydia (psittaci y thracomatis).

En los pacientes estudiados se recogió una muestra de 2-3 ml de sangre en un tubo con gelosa, que posteriormente se centrifugó, guardando los sueros a $-20^{\circ}$. En ellos se determinaron anticuerpos IgG anti-C. pneumoniae, en la Sección de Serología (Servicio de Análisis Clínicos) de nuestro hospital, utilizando la técnica de MIF, que representa la prueba de referencia internacional para detectar anticuerpos específicos frente a $C$. pneumoniae. Esta prueba está basada en la detección indirecta de anticuerpos IgG contra C. pneumoniae, con isocianato de fluoresceína como marcador de detección $^{8}$. La determinación de los anticuerpos se realiza por método en "sándwich" y consta de dos etapas; en una primera el suero del paciente se diluye en Phosphate Buffered Saline (PBS), se añade a un porta en contacto con el sustrato (antígeno), se incuba y se lava para eliminar el antígeno no unido al anticuerpo; en la segunda etapa se añade anticuerpo IgG marcado con fluoresceína que reacciona con los complejos antígeno-anticuerpo presentes, posteriormente se examina al microscopio de fluorescencia, apareciendo las reacciones positivas de color verde manzana. 
Se estableció que las muestras fueran interpretadas por el mismo médico del laboratorio. El punto de corte considerado como serología positiva lo establecimos en 1/64 basándonos en diferentes estudios ${ }^{9-13}$, que también utilizaron este mismo título como punto a partir del cual el individuo se consideraba como seropositivo frente a $C$. pneumoniae. Los pacientes fueron estratificados en dos grupos según el título de anticuerpos. Un primer grupo en el que no se detectaron anticuerpos anti-C. pneumoniae (seronegativo) y un segundo grupo con un título superior a 1/64 (seropositivo). Este título indica infección pasada.

Los parámetros analíticos de colesterol total, lipoproteína de alta densidad (HDL), lipoproteína de baja densidad (LDL) colesterol y triglicéridos, se determinaron en el laboratorio de Bioquímica por métodos enzimáticos automatizados en un autoanalizador modelo BM/Hitachi 917. Roche. Diagnostic. Los puntos de corte los situamos en los siguientes valores: para el colesterol total en $220 \mathrm{mg} / \mathrm{dl}$, HDL colesterol en $50 \mathrm{mg} / \mathrm{dl}$, LDL colesterol en $150 \mathrm{mg} / \mathrm{dl}$ y triglicéridos en $200 \mathrm{mg} / \mathrm{dl}$, según recomendaciones del Panel de Expertos del Programa Nacional de Educación en Colesterol ${ }^{14,15}$.

Paralelamente se recogieron de la historia clínica de los pacientes datos demográficos y de identificación (edad, sexo, lugar de residencia, domicilio y número de teléfono) y datos de posteriores reingresos por episodios cardiovasculares y de mortalidad de causa cardiovascular a lo largo del estudio, con una duración de tres años desde la inclusión del primer paciente. Los datos de fecha y causa de fallecimiento se obtuvieron a través de la historia clínica del paciente y del registro civil en aquellos pacientes que no fallecieron en nuestro hospital.

El análisis estadístico fue realizado en un ordenador Pentium II utilizando el programa estadístico SPSS 9.0. La prueba de Fisher o la de $\chi^{2}$ fueron usadas para analizar variables cualitativas. Para las cuantitativas se empleó la U de Mann Whitney y la supervivencia a largo plazo se evaluó mediante la prueba de Kaplan-Meier. Se aplicó un modelo de regresión logística para estudiar la relación entre mortalidad y factores de riesgo asociados (serología positiva y edad). El estudio siguió las normas del Comité de Ética del Hospital y estuvo de acuerdo con la Declaración de Helsinki.

\section{Resultados}

En el subgrupo de pacientes con infarto agudo de miocardio hubo un claro predominio de varones frente a mujeres; el rango de edad más frecuente se situó entre los 50 y 59 años y la procedencia fue fundamentalmente urbana. No hubo asociacion estadísticamente significativa entre las características demográficas (edad, sexo y procedencia) y la presencia de anticuerpos frente a C. pneumoniae. Respecto a los factores de riesgo cardiovascular estudiados (tabaco, hipertensión arterial, diabetes mellitus y perfil lipídico), tampoco observamos asociación estadísticamente significativa con la tasa de anticuerpos anti-C. pneumoniae.

En el subgrupo de pacientes con angina estable $e$ inestable las características fueron similares al grupo del infarto de miocardio, predominio de varones sobre mujeres; el rango de edad más frecuente fue en ambos casos el de 60-69 años y la procedencia fue también mayoritariamente urbana. En ninguno de los dos grupos encontramos asociación estadísticamente significativa entre las características demográficas (edad, sexo y ámbito de procedencia), así como los factores de riesgo cardiovascular (tabaco, hipertensión arterial, diabetes y perfil lipídico) y la presencia de anticuerpos frente a C. pneumoniae.

En el grupo de infarto agudo de miocardio, de los 97 pacientes en los que se determinaron anticuerpos IgG frente a $C$. pneumoniae, el 43,3\% tenían títulos positivos, superiores a $1 / 64$. Observamos una relación inversa entre la presencia de anticuerpos IgG antiC. pneumoniae y mortalidad por todas las causas, con una $p=0,02$, calculada por el estadístico exacto de Fisher. Por tanto, en nuestro grupo hay menos mortalidad entre los que tienen serología positiva (tabla 1). La supervivencia media de los pacientes con serología positiva fue de $929 \pm 21$ días y la de los pacientes con serología negativa de $800 \pm 44$ días, con una $\mathrm{p}=$ 0,007 . Al corregir los dos grupos por la edad desaparece esta significación estadística. Se realizó una regresión logística considerando mortalidad, edad y serología, siendo la edad la única variable explicativa de la mortalidad, con una $\mathrm{p}=0,0012$, perdiendo la serología su significación. Los pacientes con serología negativa tenían una supervivencia menor porque su edad media era mayor.

En el grupo de ángor inestable, de los 83 pacientes incluidos en el estudio, el 42,2\% tenían anticuerpos IgG anti-C. pneumoniae a títulos superiores a $1 / 64$. No observamos asociación, estadísticamente significativa, entre los pacientes que reingresan y la serología positiva, así como tampoco la hubo en el caso de la mortalidad en el grupo de pacientes con ángor inestable (tabla 2). La supervivencia media de los pacientes con serología positiva fue de $907 \pm 48$ días y la de los pacientes con serología negativa de $850 \pm 49$ días. No encontrando asociación, estadísticamente significativa, entre la serología y el tiempo de supervivencia de los pacientes con una $p=0,27$.

En el grupo de 69 pacientes incluidos en el estudio con angina estable, el 58\% tenía títulos de anticuerpos IgG anti-C. pneumoniae $1 / 64$ o superiores. No encontramos tampoco asociación estadísticamente significativa entre la serología a $C$. pneumoniae y los factores pronósticos de morbimortalidad (reingresos y mortalidad) en la angina estable (tabla 3).

\section{TABLA 1}

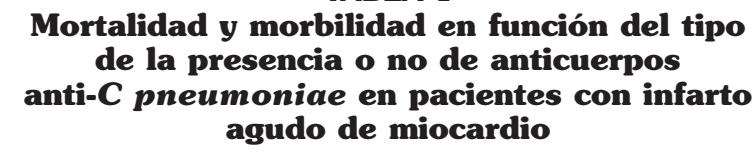

\begin{tabular}{|c|c|c|c|c|}
\hline \multirow{2}{*}{$\begin{array}{l}\text { Factores pronósticos } \\
\text { morbimortalidad }\end{array}$} & \multicolumn{4}{|c|}{ Infarto agudo de miocardio } \\
\hline & IgG+ & IgG- & $\mathbf{p}$ & IC 95\% \\
\hline \multicolumn{5}{|l|}{ Reingresos } \\
\hline Sí & $26(61,9 \%)$ & $28(50,9 \%)$ & 0,309 & $0,7-3,55$ \\
\hline \multicolumn{5}{|l|}{ Mortalidad } \\
\hline $\begin{array}{l}\text { Sí } \\
\text { No }\end{array}$ & $\begin{array}{c}2(4,8 \%) \\
40(95,2 \%)\end{array}$ & $\begin{array}{l}12(21,8 \%) \\
43(78,2 \%)\end{array}$ & 0,02 & $0,04-0,851$ \\
\hline
\end{tabular}

Observamos una relación inversa entre la presencia de anticuerpos IgG antiC. pneumoniae y mortalidad por todas las causas, con una $\mathrm{p}=0,02 \mathrm{calcu}-$ lada por el estadístico exacto de Fisher. 
TABLA 2

Mortalidad y morbilidad en función del tipo
de la presencia o no de anticuerpos
anti-C pneumoniae en pacientes con angina
inestable

\begin{tabular}{c|c|c|c|c}
\hline \multirow{2}{*}{$\begin{array}{c}\text { Factores pronósticos } \\
\text { morbimortalidad }\end{array}$} & \multicolumn{4}{|c}{ Angina inestable } \\
\cline { 2 - 5 } & IgG+ & IgG- & p & IC 95\% \\
\hline
\end{tabular}

\begin{tabular}{lrlll} 
Reingresos & & & & \\
Sí & $18(51,4 \%)$ & $24(50 \%)$ & 1 & $0,44-2,53$ \\
$\quad$ No & $17(48,6 \%)$ & $24(50 \%)$ & & \\
Mortalidad & $4(11,4 \%)$ & $10(20,8 \%)$ & 0,375 & $0,14-1,716$ \\
$\quad$ Sí & $31(88,6 \%)$ & $38(79,2 \%)$ & & \\
No & & & \\
\hline
\end{tabular}

La supervivencia media de los pacientes con serología positiva fue de $894 \pm 38$ días y la de los pacientes con serología negativa de $874 \pm 27$ días. No hubo asociación, estadísticamente significativa, entre la serología y la supervivencia de los pacientes con una $\mathrm{p}=0,21$.

\section{Discusión}

Desde mediados de los años ochenta se ha relacionado a la C. pneumoniae con la arteriosclerosis coronaria. Son numerosos los estudios que hasta la actualidad se han publicado intentando establecer una asociación causal entre este microorganismo y la cardiopatía isquémica sin llegar a confirmarse este punto, pero sí a demostrar en muchos de esos trabajos, asociación epidemiológica entre anticuerpos anti-C. pneumoniae y aumento del riesgo de enfermedad coronaria.

En nuestro grupo de pacientes con infarto agudo de miocardio la prevalencia de anticuerpos frente a C. pneumoniae fue del $43,3 \%$, resultado similar al obtenido por Ridker et al ${ }^{16}$ en un grupo de 343 pacientes con infarto agudo de miocardio $(41,7 \%)$ que posteriormente siguieron durante doce años. Ellos no encontraron que la seropositividad $\operatorname{IgG}$ a $C$. pneumoniae sea un marcador de riesgo futuro de infarto de miocardio. Sin embargo, autores como Davidson et $\mathrm{al}^{17}$ sí observaron que los títulos de IgG y no los de

TABLA 3

\begin{tabular}{|c|c|c|c|c|}
\hline \multicolumn{5}{|c|}{$\begin{array}{c}\text { Mortalidad y morbilidad en función del tipo } \\
\text { de la presencia o no de anticuerpos } \\
\text { anti-C pneumoniae en pacientes con angina } \\
\text { estable }\end{array}$} \\
\hline \multirow{2}{*}{$\begin{array}{l}\text { actores pronósticos } \\
\text { morbimortalidad }\end{array}$} & \multicolumn{4}{|c|}{ Angina estable } \\
\hline & IgG+ & IgG- & $\mathbf{p}$ & IC 95\% \\
\hline \multirow{3}{*}{$\begin{array}{l}\text { Reingresos } \\
\text { Sí } \\
\text { No } \\
\text { Mortalidad } \\
\text { Sí } \\
\text { No }\end{array}$} & & & \multirow[b]{2}{*}{0,789} & \multirow[b]{2}{*}{$0,4-3,57$} \\
\hline & $\begin{array}{l}11(27,5 \%) \\
29(72,5 \%)\end{array}$ & $\begin{array}{c}7(24 \%) \\
22(75,9 \%)\end{array}$ & & \\
\hline & $\begin{array}{c}2(5 \%) \\
38(95 \%)\end{array}$ & $\begin{array}{c}2(6,9 \%) \\
27(93,1 \%)\end{array}$ & 1 & $0,09-5,36$ \\
\hline
\end{tabular}

IgA o IgM se correlacionaban con la capacidad de detectar C. pneumoniae dentro de las arterias coronarias.

Otros autores encontraron prevalencias más altas de anticuerpos IgG en el infarto agudo de miocardio como Blasi et al ${ }^{18}$ en un grupo de 61 pacientes con un $57,3 \%$ de seropositividad y hasta un $89,6 \%$ de prevalencia en el infarto que obtuvieron Mazzoli et $\mathrm{al}^{8}$. En ambos casos se consideró positivo títulos de IgG $>1 / 16$, lo que probablemente incrementó aún más la prevalencia.

En nuestro estudio observamos una relación inversa entre la seropositividad frente a C. pneumoniae y la mortalidad de los pacientes en un período de tres años posteriores a su inclusión en el estudio, con una $p=0,01$. Sin embargo, al realizar una regresión logística, incluyendo la edad, desaparecía esta significación. Nuestros pacientes con serología negativa tenían una supervivencia menor porque su edad era mayor. La edad constituye un importante factor pronóstico en la cardiopatía isquémica.

En el caso de los reingresos no encontramos asociación con la serología. Son escasos los trabajos publicados que relacionan la serología a $C$. pneumoniae con el riesgo de mortalidad por cardiopatía isquémica. Uno de los primeros estudios en esta línea fue el llevado a cabo por Strachan et al ${ }^{19}$ de manera prospectiva durante 13 años en Caerphilly, área de South Wales, determinando anticuerpos IgG e IgA y observando asociación bordeline significativa, $p=0,048$, entre títulos de IgG y mortalidad por todas las causas, mientras que con los anticuerpos IgA se asociaban con un significativo aumento del riesgo de mortalidad (OR: 1,47; intervalo de confianza del 95\% de 1,12 a $1,94)$. También observaron en este trabajo que los anticuerpos IgG no se relacionaban significativamente con la incidencia y la prevalencia de enfermedad cardíaca isquémica, mientras que en el caso de los anticuerpos IgA sí había asociación con prevalencia de cardiopatía isquémica (OR: 1,49; intervalo de confianza del $95 \%$ de 1,16 a 1,92), pero no con incidencia (OR: 1,14; intervalo de confianza del 95\% de 0,84 a 1,56). En nuestro estudio no se determinaron anticuerpos IgA.

Por el contrario, el estudio finlandés ${ }^{20}$, con un seguimiento de 6 años, no encuentra relación entre anticuerpos frente a $C$. pneumoniae, IgG e IgA a títulos $>1 / 32$ y muerte por cardiopatía isquémica. Únicamente en varones la elevación de IgG era un factor de riesgo independiente para muerte por enfermedad cerebrovascular. Nuestro estudio muestra una menor mortalidad en los pacientes con infarto de miocardio e IgG positiva a C. pneumoniae; sin embargo, al ajustar por la edad desaparece esta significación, ya que la media de edad de los pacientes con serología negativa es mayor que la de los pacientes con serología positiva. El segundo pico de máxima incidencia de infección por C. pneumoniae se produce alrededor de los 70 años, con una duración media de los anticuerpos IgG entre 3 y 5 años, por tanto a partir de los 7580 años se produciría un descenso de dichos anticuerpos $^{21,22}$. El corte para determinar la positividad de 
los anticuerpos IgG anti-C. pneumoniae es similar al utilizado por el grupo de Saikku y nuestro seguimiento es únicamente de tres años.

En nuestros resultados obtuvimos una prevalencia de anticuerpos IgG frente a C. pneumoniae del $42,2 \%$ en los pacientes con angina inestable. No encontramos en este grupo de pacientes asociación entre la seropositividad a $C$. pneumoniae y los factores pronósticos de morbimortalidad, reingresos y mortalidad en los tres años posteriores a la inclusión de los pacientes en el estudio. Como citamos en el grupo del infarto agudo de miocardio, sí había asociación bordeline $\mathrm{p}<0,048$ entre los anticuerpos IgG y la mortalidad por todas las causas en un estudio llevado a cabo por Strachan et $\mathrm{al}^{19}$ en la cardiopatía isquémica. En nuestros resultados, en el grupo de pacientes con angina estable la prevalencia de anticuerpos IgG frente a $C$. pneumoniae fue del $58 \%$. Este dato contrasta con la prevalencia encontrada por Hoffmeister et $\mathrm{al}^{10}$ en una población alemana, que fue del $87,4 \%$, llevado a cabo en pacientes con enfermedad arterial coronaria estable para investigar la asociación entre la seropositividad a $C$. pneumoniae y a lipopolisacárido Chlamydial y la enfermedad coronaria documentada angiográficamente.

Al igual que en el grupo de la angina inestable, no hubo asociación de los factores pronósticos de morbimortalidad (reingresos y mortalidad) con la serología positiva.

En contraste el grupo de Strachan et $\mathrm{al}^{19}$, sí encontró asociación de la mortalidad en pacientes con cardiopatía isquémica y seropositividad IgG a C. pneumoniae.

Los estudios realizados in vitro relacionan la infección por C. pneumoniae y la presencia de su proteína HSP 60 no solamente con la formación de la placa de ateroma, sino con la inestabilidad de la misma, lo que deberá determinar un aumento de la morbimortalidad. Se han implicado diversos mecanismos: inducción de factor de necrosis tumoral- $\alpha$ (TNF- $\alpha$ ), producción de metaloproteasas, inducción de moléculas de adhesión e incremento de la producción de IL-6. Sin embargo, estos resultados no se corresponden con la realidad clínica. Una posible explicación es la utilización de estatinas en el tratamiento posterior del infarto de miocardio. Nuestros pacientes tenían niveles bajos de colesterol y triglicéridos, lo que parece indicar un uso amplio de las mismas. Algunas de estas estatinas a dosis altas son capaces de inhibir el complejo NF- $\mathrm{KB}^{23}$, que es responsable de la producción de IL-6 y de la inducción de moléculas de adhesión por la infección por Chlamydia. Esta inhibición podría explicarnos la ausencia de relación.

No hemos encontrado relación entre la presencia de anticuerpos IgG frente a C. pneumoniae y morbimortalidad en la población con cardiopatía isquémica de nuestra área. Son resultados similares a los publicados en la literatura ${ }^{24-27}$, la presencia de anticuerpos IgG e IgA no se asoció a un mayor riesgo de muerte. Podemos concluir que la presencia de anticuerpos IgG frente a C. pneumoniae no es un marcador de morbimortalidad en nuestra población.

\section{BIBLIOGRAFÍA}

1. Gupta S, Camm AJ. Chlamydia pneumoniae and coronary heart disease. Coincidence, association, or causation? BMJ 1997;314:1778-9.

2. Sumpter MT, Dunn MI. Is coronary artery disease an Infectious disease? CHEST 1997;112:301-3.

3. Gurfinkel EP, Seirica BM, Bozovich G. Aterosclerosis e infección ¿estamos olvidando algo? En: Picazo JJ, Bouza E, editores. Infección 1999. Bil bao: Servisistem, 2000; p. 93-122.

4. Saikku P, Mattila K, Nieminen MS, Huttunen JK, Leinonen M, Ekman MR et al. Serological evidence of an association of a novel Chlamydia. TWAR, with chronic coronary heart disease and acute myocardial infarction. Lancet 1988;2:983-6.

5. Thomas M, Wong Y, Thomas D, Ajaz M, Tsang V, Gallagher PJ, et al. Relation between direct detection of Chlamydia pneumoniae DNA in human coronary arteries at postmortem examination and histological severity (stary grading) of associated atherosclerotic plaque. Circulation 1999;99: 2733- 6

6. Esposito G, Blasi F, Allegra L, Chiesa R, Melissano G, Cosentini R, et al. Demonstration of viable Chlamydia pneumoniae in atherosclerotic plaques of carotid arteries by reverse transcriptase polymerase chain reaction. Ann Vasc Surg 1999;13:421-5.

7. Azpitarte Almagro J, Cabadés O'Callaghan A, López Merino V, de los Reyes López M, San José Garagarza JM. Angina de pecho. Concepto y clasificación. Rev Esp Cardiol 1995;48:373-82.

8. Mazzoli S, Tofani N, Fantini A, Semplici F, Bandini F, Salvi A, et al. Chlamydia pneumoniae antibody response in patients with acute myocardial infarction and their follow-up. American Heart Journal, 1998;125:15-20.

9. Nieto FJ, Folson AR, Sorlie PD, Grayston JT, Wang SP, Chambless LE. Chlamydia pneumoniae infection and incident coronary heart disease: the atherosclerosis Risk in Comunities Study. Am J Epidemiol 1999;150:149-56. 10. Hoffmeister A, Rothenbacher DP, Wanner P, Bode G, Persson K Brenner HE, et al. Seropositivity to Chlamydia lipopolysaccharide and Chlamydia pneumoniae, systemic inflammation and stable coronary artery disease. JACC 2000;35:115-8.

11. Gufinkel E, Bozovich G, Beck E, Testa E, Livellara D, Mautner B. Treatment with the antibiotic roxithromycin in patients with acute non-Q-wave coronary syndromes. The final report of the ROXIS study. Eur Heart J 1999; 20:121-7.

12. Änestad G, Scheel O, Hungnes O. Chronic infections and coronary heart disease. Lancet 1997;350:1028-30.

13. Gupta S, Leatham EW, Carrington D, Mendall MA, Kaski JC, Camm AJ. Elevated Chlamydia pneumoniae antibodies, cardiovascular events, and azithromycin in male survivors of myocardial infarction. Circulation 1997;96:404-7. 14. Grundy SM, Bilheimer D, Chait A, Clark LT, Denke M, Havel RJ. Summary of the second report of the National Cholesterol Education Program (NCEP) expert panel on detection, evaluation, and treatment of high blood cholesterol in adults (adult treatment panel II). JAMA 1993;269:3015-23. 15. Rapaport E, Bilheimer DW, Chobanian AV, Hajjar DP, Hawkins CM Hutchins GM. Triglyceride, high-density lipoprotein, and coronary heart disease. JAMA 1993;269:505-10.

16. Ridker PM, Kundsin RB, Stampfer MJ, Poulin S, Hennekens CH. Prospective study Chlamydia pneumoniae IgG seropositivity and risk of future myocardial infarction. Circulation 1999;99:1161-4.

17. Davidson M, Kuo CC, Middaugh JP, Campbell LA, Wang SP, Newman WP et al. Confirmed previous infection with Chlamydia pneumoniae (TWAR) and its presence in early coronary atherosclerosis. Circulation 1998;98:628-33.

18. Blasi F, Consentini R, Raccanelli R, Massari FM, Arosio C, Tarsia P, et al. A possible association of Chlamydia pneumoniae infection and acute myocardial infarction in patients younger than 65 years of age. CHEST 1997; 112:309-12

19. Strachan DP, Carrington D, Mendall MR, Ballam L, Morris J, Butland BK, et al. Relation of Chlamydia pneumoniae serology to mortality and inci dence of ischaemic heart disease over 13 years in the Caerphilly prospective heart disease study. BMJ 1999;318:1035-40.

20. Herzen LV, Isoaho R, Kivelä SL, Saikku P. Relation of C. pneumoniae antibodies to ischaemic heart disease. Finnish study finds significant association between raised IgG, but not IgA, titres and mortality. BMJ 1999;319 1575 .

21. Álvarez-Sala JL, García-Casasola G. Infección por Chlamydia pneumoniae. Medicina Interna. Farreras Rozman. 1995; Vol. II. 13. a ed., 2414-17. 22. Cobos J, Fuentes F, Ruiz G, Prieto J. Procedimientos diagnósticos en enfermedades causadas por Chlamydia pneumoniae. Rev Clin Esp 1998; 198:12-8.

23. Ortego M, Bustos C, Hernández-Presa MA, Tunon J, Díaz C, Hernán $\operatorname{dez} \mathrm{G}$, et al. Atorvastatin reduces NF-KappaB activation and chemokine espression in vascular smooth muscle cells and mononuclear cells. Atherosclerosis $1999 ; 147: 253-61$.

24. Siscovick DS, Schwartz SM, Corey L, Grayston JT, Ashley R, Wang SP, et al. Chlamydia pneumoniae, herpes simplex virus type 1 , and cytomegalovitus and incident myocardial infarction and coronary heart disease death in older adults; the Cardiovascular Health Study. Circulation 2000; 7:2335-40.

25. Zhu J, Nieto FJ, Horne BD, Anderson JL, Muhlestein JB, Epstein SE. Prospective study of pathogen burden and risk of myocardial infarction or death. Circulation 2001;103:45-51.

26. Rupprecht HJ, Blakenberg S, Bickel C, Rippin C, Hafner G, Prellwitz W, et al. Impact of viral and bacterial infectious burden on long-tern prognosis in patients with coronary artery disease. Circulation 2001;104:35-1.

27. Wald NJ, Law MR, Morris JK, Zhou X, Wong Y, Ward ME. Chlamydia pneumoniae infection and mortality from ischaemic heart disease: large prospective study. BMJ 2000;321:187-8. 